\title{
Single and Multivalued Maps on Parametric Metric Spaces Endowed with an Equivalence Relation
}

\author{
Muhammad Usman Ali $\mathbb{D}^{1},{ }^{1}$ Hassen Aydi $\left(\mathbb{D},{ }^{2,3,4}\right.$ Asma Batool, ${ }^{1}$ Vahid Parvaneh ${ }^{(D)}{ }^{5}$ \\ and Naeem Saleem ${ }^{6}{ }^{6}$ \\ ${ }^{1}$ Department of Mathematics, COMSATS University Islamabad, Attock Campus, Attock, Pakistan \\ ${ }^{2}$ Université de Sousse, Institut Supérieur d'Informatique et des Techniques de Communication, H. Sousse, 4000, Tunisia \\ ${ }^{3}$ Department of Mathematics and Applied Mathematics, Sefako Makgatho Health Sciences University, Ga-Rankuwa, South Africa \\ ${ }^{4}$ China Medical University Hospital, China Medical University, Taichung 40402, Taiwan \\ ${ }^{5}$ Department of Mathematics, Gilan-E-Gharb Branch, Islamic Azad University, Gilan-E-Gharb, Iran \\ ${ }^{6}$ Department of Mathematics, University of Management and Technology, Lahore, Pakistan
}

Correspondence should be addressed to Hassen Aydi; hassen.aydi@isima.rnu.tn and Naeem Saleem; naeem.saleem2@gmail.com

Received 4 July 2021; Accepted 27 December 2021; Published 28 January 2022

Academic Editor: Sergey Shmarev

Copyright (C) 2022 Muhammad Usman Ali et al. This is an open access article distributed under the Creative Commons Attribution License, which permits unrestricted use, distribution, and reproduction in any medium, provided the original work is properly cited.

\begin{abstract}
This article presents the E-parametric metric space, which is a generalized concept of parametric metric space. After that, the discussion is concerned with the existence of fixed points of single and multivalued maps on E-parametric metric spaces satisfying some contractive inequalities defined by an auxiliary function.
\end{abstract}

\section{Introduction and Preliminaries}

Metric fixed point theory is a very fruitful area of research belonging to nonlinear analysis and operator theory. It has much usage in other fields of mathematics. Numerous generalizations of Banach contraction principle have flourished this field in several aspects through using generalized forms of metric spaces or contraction conditions. It is not wrong to say that the contraction condition given by Salimi et al. in [1] is the most renowned condition of this decade, whereas on the account of metric spaces, we have many abstract forms like $b$-metric spaces [2-5], partial metric spaces [6, $7]$, and partial $b$-metric spaces $[8,9]$. Recently, the notions of $R$-metric [10] and parametric metric spaces [11] have been initiated.

In the following, we recall the concept of parametric metric spaces [11] and the convergence of sequences.

A map $P_{m}: H \times H \times(0, \infty) \longrightarrow[0, \infty)($ where $H \neq \varnothing)$ is said to be a parametric metric on $H$ if it satisfies the following axioms: (i) $P_{m}\left(\ell, \ell^{\prime}, \curlyvee\right)=0$ for all $\vee>0 \Longleftrightarrow \ell=\ell^{\prime}$

(ii) $P_{m}\left(\ell, \ell^{\prime}, \curlyvee\right)=P\left(\ell^{\prime}, \ell, \curlyvee\right)$ for all $\curlyvee>0$

(iii) $P_{m}\left(\ell, \ell^{\prime}, \curlyvee\right) \leq P_{m}\left(\ell, \ell^{\prime \prime}, \curlyvee\right)+P_{m}\left(\ell^{\prime \prime}, \ell^{\prime}, \curlyvee\right)$ for all $\ell$, $\ell^{\prime}, \ell^{\prime \prime} \in H$ and for all $\vee>0$

Then, $\left(H, P_{m}\right)$ is a parametric metric space. If $\left(H, P_{m}\right)$ be a parametric metric space and $\left\{\ell_{n}\right\} \subseteq H$, then

(1) $\ell_{n} \longrightarrow \ell \in H \Longleftrightarrow \lim _{n \rightarrow \infty} P_{m}\left(\ell_{n}, \ell, \curlyvee\right)=0$ for all $\curlyvee>0$

(2) $\left\{\ell_{n}\right\}$ is Cauchy if $\lim _{m, n \longrightarrow \infty} P_{m}\left(\ell_{m}, \ell_{n}, \curlyvee\right)=0$ for all $\curlyvee>0$

Note that $\left(H, P_{m}\right)$ is complete if each Cauchy sequence in $H$ converges in $H$.

One of the ways to generalize the contraction condition is to define it via some auxiliary functions. For example, an auxiliary type function was given in [12], where $Q_{\xi}$ represents the collection of functions $Q:\left(\mathbb{R}^{+}\right)^{4} \longrightarrow \mathbb{R}^{+}=[0, \infty)$ satisfying the below axioms: 
(i) $Q$ is nondecreasing and continuous with respect to its each coordinate

(ii) If $q_{1}, q_{2} \in \mathbb{R}^{+}$with $q_{1}<q_{2}$ and $q_{1} \leq Q\left(q_{2}, q_{2}, q_{1}, q_{2}\right)$, then $q_{1} \leq \xi\left(q_{2}\right)$;

(iii) If $q_{1}, q_{2} \in \mathbb{R}^{+}$with $q_{1} \geq q_{2}$ and $q_{1} \leq Q\left(q_{1}, q_{2}, q_{1}, q_{1}\right)$, then $q_{1}=0$

(iv) If $q \in \mathbb{R}^{+}$with $q \leq Q(0,0, q,(1 / 2) q)$ or $q \leq Q(0, q$, $0,(1 / 2) q)$ or $q \leq Q(q, 0,0, q)$, then $q=0$

and $\xi: \mathbb{R}^{+} \longrightarrow \mathbb{R}^{+}$is a nondecreasing map with $\sum_{n=1}^{\infty} \xi^{n}(t)$ $<\infty$ for all $t \geq 0$ and $\xi(t)<t$ for all $t>0$.

As examples of $Q \in Q_{\xi}$, we cite the following:

(i) Let $Q_{1}\left(q_{1}, q_{2}, q_{3}, q_{4}\right)=k \max \left\{q_{1}, q_{2}, q_{3}, q_{4}\right\}$ with $\xi$ $(t)=k t$, where $k \in[0,1)$.

(ii) Let $Q_{2}\left(q_{1}, q_{2}, q_{3}, q_{4}\right)=k q_{4}$ with $\xi(t)=k t$, where $k$ $\in[0,1)$.

(iii) Let $Q_{3}\left(q_{1}, q_{2}, q_{3}, q_{4}\right)=a^{*} q_{1}+b^{*} q_{2}+c^{*} q_{4}$ with $\xi(t)$ $=\left(a^{*}+b^{*}+c^{*}\right) t$, where $a^{*}, b^{*}, c^{*} \geq 0$ and $a^{*}+b^{*}$ $+c^{*} \in[0,1)$.

Khalehoghli et al. [10] took a binary relation $E$ on $H$ and a simple metric $d$ on $H$ to define an $R$-metric space, denoted as $\left(H, d, B_{R}\right)$. They also said that a self map $T$ on $H$ is a $B_{R}$ -contraction, if

$$
d\left(T \ell, T \ell^{\prime}\right) \leq k d\left(\ell, \ell^{\prime}\right)
$$

for all $\ell, \ell^{\prime} \in H$ with $\ell B_{R} \ell^{\prime}$, where $0<k<1$. Along with this definition, the authors also defined the concepts of $B_{R}$-continuity and $B_{R}$-preserving property in order to extend the result of Banach on $R$-metric spaces.

\section{Main Results}

Let $H \neq \varnothing$ and $E$ be an equivalence relation on $H$. We introduce the following: a function $P_{m}: H \times H \times(0, \infty) \longrightarrow[0$, $\infty)$ is an $E$-parametric-metric on $H$, provided that the following axioms hold: (i) $P_{m}\left(\ell, \ell^{\prime}, \curlyvee\right)=0 \Longleftrightarrow \ell=\ell^{\prime}$ for all $\ell \sim \ell^{\prime}$ and for all $\vee>0$

(ii) $\left(P_{m}\left(\ell, \ell^{\prime}, \vee\right)=P_{m}\left(\ell^{\prime}, \ell, \vee\right)\right.$ for all $\vee>0$ and for all $\ell \sim \ell^{\prime}$

(iii) $P_{m}\left(\ell, \ell^{\prime}, \vee\right) \leq P_{m}\left(\ell, \ell^{\prime \prime}, \vee\right)+P_{m}\left(\ell^{\prime \prime}, \ell^{\prime}, \vee\right)$ for all $\ell, \ell^{\prime}$, $\ell^{\prime \prime} \in H$, for all $\vee>0$ and for all $\ell \sim \ell^{\prime} \sim \ell^{\prime \prime}$

Then, $\left(H, E, P_{m}\right)$ is an $E$-parametric metric space.

Example 1. Take $H$ as the collection of all functions $g:(0$, $\infty) \longrightarrow R$. Consider an equivalence relation $E$ on $H$ defined by $g E f$ if $t \mid f(t)-g(t)$ for all $t \in(0, \infty)$ (note that $\mid$ means that $t$ divides $f(t)-g(t))$. Define $P_{m}: H \times H \times(0, \infty)$ $\longrightarrow[0, \infty)$ by

$$
P_{m}(g, f, \vee)=|g(\vee)-f(\vee)| \text {, for all } g, f \in H \text { and for all } \vee>0 \text {. }
$$

Then, $\left(H, E, P_{m}\right)$ is an $E$-parametric metric space.

Below, $\left(H, E, P_{m}\right)$ stands for an $E$-parametric metric space.

Definition 1. Let $\left\{\kappa_{m}\right\}$ be an $E$-sequence in $\left(H, E, P_{m}\right)$, that is, $\kappa_{m} \sim \kappa_{m+1}$ for each $m \in \mathbb{N}$. Then, we say that

(i) $\left\{\kappa_{m}\right\}$ is a convergent sequence in $H$ and converges to $\kappa \in H$ if $\lim _{m \rightarrow \infty} P_{m}\left(\kappa_{m}, \kappa, \curlyvee\right)=0$ for all $\vee>0$ and $\kappa_{m} \sim \kappa$ for each $m \geq k$ (for some value of $k$ )

(ii) $\left\{\kappa_{m}\right\}$ is Cauchy if $\lim _{m, n \rightarrow \infty} P_{m}\left(\kappa_{m}, \kappa_{n}, \vee\right)=0$ for all $\vee>0$.

Definition 2. $\left(H, E, P_{m}\right)$ is $E$-complete if each Cauchy $E$ -sequence in $H$ is convergent in $H$.

Definition 3. $\left(H, E, P_{m}\right)$ is called regular whenever if $P_{m}(\ell$, $\left.\ell^{\prime}, \vee^{0}\right)=0$ for some $\vee^{0}>0$, then we have $P_{m}\left(\ell, \ell^{\prime}, \curlyvee\right)=0$ for all $\vee$.

We now present the first main theorem of the section.

Theorem 4. Let $\left(H, E, P_{m}\right)$ be an E-complete regular $E$ -parametric metric space. Let $T: H \longrightarrow H$ and $\alpha: H \times H$ $\longrightarrow[0, \infty)$ be two maps such that

$$
\alpha\left(\ell, \ell^{\prime}\right) P_{m}\left(T \ell, T \ell^{\prime}, \vee\right) \leq Q\left(P_{m}\left(\ell, \ell^{\prime}, \vee\right), P_{m}(\ell, T \ell, \vee), P_{m}\left(\ell^{\prime}, T \ell^{\prime}, \vee\right), \frac{P_{m}\left(\ell^{\prime}, T \ell, \vee\right)+P_{m}\left(\ell, T \ell^{\prime}, \curlyvee\right)}{2}\right),
$$

for all $\vee>0$ and for all $\ell, \ell^{\prime} \in H$ with $\ell \sim \ell^{\prime}$, where $Q \in Q_{\xi}$. Also, consider the following assertions:

(a) $T$ is E-preserving, that is, $\ell \sim \ell^{\prime}$ implies $T \ell \sim T \ell^{\prime}$

(b) There is $\ell_{0} \in H$ such that $\ell_{0} \sim T \ell_{0}$ and $\alpha\left(\ell_{0}, T \ell_{0}\right) \geq 1$ (c) For every $\ell, \ell^{\prime} \in H$ such that $\ell \sim \ell^{\prime}$ and $\alpha\left(\ell, \ell^{\prime}\right) \geq 1$, we have $\alpha\left(T \ell, T \ell^{\prime}\right) \geq 1$

(d) For each E-sequence $\left\{\ell_{m}\right\}$ in $H$ with $\alpha\left(\ell_{m}, \ell_{m+1}\right) \geq 1$ for all $m \in \mathbb{N}$ and $\ell_{m} \longrightarrow \ell$, we have $\alpha\left(\ell_{m}, \ell\right) \geq 1$ for all $m \in \mathbb{N}$ 
Then, $T$ has a fixed point.

Proof. By (b), there must be $\kappa_{0} \in H$ of the form $\kappa_{0} \sim T \kappa_{0}$ and $\alpha\left(\kappa_{0}, T \kappa_{0}\right) \geq 1$. Since $T$ is $E$-preserving, we have $T \kappa_{0} \sim T^{2} \kappa_{0}$, and by (c), $\alpha\left(T \kappa_{0}, T^{2} \kappa_{0}\right) \geq 1$. The repetition of these steps yields that $T^{m} \kappa_{0} \sim T^{m+1} \kappa_{0}$ and $\alpha\left(T^{m} \kappa_{0}, T^{m+1} \kappa_{0}\right) \geq 1$ for all $m$ $\in \mathbb{N}$. Take a sequence $\left\{\kappa_{m}\right\}$ with the terms as $\kappa_{m}=T^{m} \kappa_{0}$ for all $m \in \mathbb{N}$. Thus, $\kappa_{m} \sim \kappa_{m+1}$ and $\alpha\left(\kappa_{m}, \kappa_{m+1}\right) \geq 1$ for all $m \in$ $\mathbb{W}=\mathbb{N} \cup\{0\}$. Before doing the next part of the proof, we take $\kappa_{m} \neq \kappa_{m+1}$ for all $m \in \mathbb{W}$. From (3), for each $m \in \mathbb{W}$, we get

$$
\begin{aligned}
P_{m}\left(T \kappa_{m}, T \kappa_{m+1}, \curlyvee\right) & \leq \alpha\left(\kappa_{m}, \kappa_{m+1}\right) P_{m}\left(T \kappa_{m}, T \kappa_{m+1}, \curlyvee\right) \\
& \leq Q\left(P_{m}\left(\kappa_{m}, \kappa_{m+1}, \curlyvee\right), P_{m}\left(\kappa_{m}, T \kappa_{m}, \curlyvee\right), P_{m}\left(\kappa_{m+1}, T \kappa_{m+1}, \curlyvee\right), \frac{1}{2}\left(P_{m}\left(\kappa_{m+1}, T \kappa_{m}, \curlyvee\right)+P_{m}\left(\kappa_{m}, T \kappa_{m+1}, \curlyvee\right)\right)\right) \\
& =Q\left(P_{m}\left(\kappa_{m}, \kappa_{m+1}, \curlyvee\right), P_{m}\left(\kappa_{m}, \kappa_{m+1}, \curlyvee\right), P_{m}\left(\kappa_{m+1}, \kappa_{m+2}, \curlyvee\right), \frac{1}{2}\left(P_{m}\left(\kappa_{m+1}, \kappa_{m+1}, \curlyvee\right)+P_{m}\left(\kappa_{m}, \kappa_{m+2}, \curlyvee\right)\right)\right) \\
& \leq Q\left(P_{m}\left(\kappa_{m}, \kappa_{m+1}, \curlyvee\right), P_{m}\left(\kappa_{m}, \kappa_{m+1}, \curlyvee\right), P_{m}\left(\kappa_{m+1}, \kappa_{m+2}, \curlyvee\right), \frac{1}{2}\left(P_{m}\left(\kappa_{m}, \kappa_{m+1}, \curlyvee\right)+P_{m}\left(\kappa_{m+1}, \kappa_{m+2}, \curlyvee\right)\right)\right),
\end{aligned}
$$

for all $\curlyvee>0$.

Here, our claim is, for each $\vee>0,\left\{P_{m}\left(\kappa_{m}, \kappa_{m+1}, \curlyvee\right)\right\}_{m \in \mathbb{W}}$ is a strictly decreasing sequence. Suppose this is not true in general, then we have at least one $m_{0} \in \mathbb{W}$ such that $P_{m}$ $\left(\kappa_{m_{0}}, \kappa_{m_{0}+1}, \vee^{0}\right) \leq P_{m}\left(\kappa_{m_{0}+1}, \kappa_{m_{0}+2}, \vee^{0}\right)$ for some $\vee^{0}>0$. Then, from (4) and property (i) of $Q_{\xi}$, we get

$$
\begin{aligned}
P_{m}\left(\kappa_{m_{0}+1}, \kappa_{m_{0}+2}, \vee^{0}\right) & =P_{m}\left(T \kappa_{m_{0}}, T \kappa_{m_{0}+1}, \vee^{0}\right) \\
& \leq Q\left(P_{m}\left(\kappa_{m_{0}}, \kappa_{m_{0}+1}, \vee^{0}\right), P_{m}\left(\kappa_{m_{0}}, \kappa_{m_{0}+1}, \vee^{0}\right), P_{m}\left(\kappa_{m_{0}+1}, \kappa_{m_{0}+2}, \vee^{0}\right), \frac{1}{2}\left(P_{m}\left(\kappa_{m_{0}}, \kappa_{m_{0}+1}, \vee^{0}\right)+P_{m}\left(\kappa_{m_{0}+1}, \kappa_{m_{0}+2}, \vee^{0}\right)\right)\right) \\
& \leq Q\left(P_{m}\left(\kappa_{m_{0}+1}, \kappa_{m_{0}+2}, \vee^{0}\right), P_{m}\left(\kappa_{m_{0}}, \kappa_{m_{0}+1}, \vee^{0}\right), P_{m}\left(\kappa_{m_{0}+1}, \kappa_{m_{0}+2}, \vee^{0}\right), P_{m}\left(\kappa_{m_{0}+1}, \kappa_{m_{0}+2}, \vee^{0}\right)\right) .
\end{aligned}
$$

Property (iii) of $Q_{\xi}$ together with the above inequality implies that

$$
P_{m}\left(\kappa_{m_{0}+1}, \kappa_{m_{0}+2}, \vee^{0}\right)=0, \text { for } \curlyvee^{0}>0 .
$$

This fact and regularity condition give us

$$
P_{m}\left(\kappa_{m_{0}+1}, \kappa_{m_{0}+2}, \vee\right)=0 \text {, for all } \vee>0,
$$

which is not possible under the assumption. Thus, $P_{m}\left(\kappa_{m}\right.$, $\left.\kappa_{m+1}, \curlyvee\right)>P_{m}\left(\kappa_{m+1}, \kappa_{m+2}, \curlyvee\right)$ for all $\curlyvee>0$ and $m \in \mathbb{W}$. Again, we use (4) and property (i) of $Q_{\xi}$ to obtain

$$
\begin{aligned}
P_{m}\left(\kappa_{m+1}, \kappa_{m+2}, \curlyvee\right)= & P_{m}\left(T \kappa_{m}, T \kappa_{m+1}, \curlyvee\right) \\
\leq & Q\left(P_{m}\left(\kappa_{m}, \kappa_{m+1}, \curlyvee\right), P_{m}\left(\kappa_{m}, \kappa_{m+1}, \curlyvee\right), P_{m}\right. \\
& \left.\cdot\left(\kappa_{m+1}, \kappa_{m+2}, \curlyvee\right), P_{m}\left(\kappa_{m}, \kappa_{m+1}, \curlyvee\right)\right),
\end{aligned}
$$

for all $\vee>0$ and $m \in \mathbb{W}$.
Property (ii) of $Q_{\xi}$ together with (8) implies

$P_{m}\left(\kappa_{m+1}, \kappa_{m+2}, \curlyvee\right) \leq \xi\left(P_{m}\left(\kappa_{m}, \kappa_{m+1}, \curlyvee\right)\right)$, for all $\vee>0$ and $m \in \mathbb{W}$.

After a few simplification, we get

$$
P_{m}\left(\kappa_{m}, \kappa_{m+1}, \curlyvee\right) \leq \xi^{m}\left(P_{m}\left(\kappa_{0}, \kappa_{1}, \curlyvee\right)\right) \text {, for all } \curlyvee>0 \text { and } m \in \mathbb{N} \text {. }
$$

Hence, $\left\{\kappa_{m}\right\}$ is a sequence in $H$ with $\kappa_{m} \sim \kappa_{m+1}$ and $P_{m}$ $\left(\kappa_{m}, \kappa_{m+1}, \curlyvee\right) \leq \xi^{m}\left(P_{m}\left(\kappa_{0}, \kappa_{1}, \curlyvee\right)\right)$ for all $\curlyvee>0$ and $m \in \mathbb{N}$. Now, we will show that this $E$-sequence is also Cauchy. For each $n>m \in \mathbb{N}$, we can obtain

$P_{m}\left(\kappa_{m}, \kappa_{n}, \curlyvee\right) \leq \sum_{j=m}^{n-1} P_{m}\left(\kappa_{j}, \kappa_{j+1}, \curlyvee\right) \leq \sum_{j=m}^{\infty} \xi^{j}\left(P_{m}\left(\kappa_{0}, \kappa_{1}, \curlyvee\right)\right)$, for all $\curlyvee>0$.

The convergence of $\sum_{j=1}^{\infty} \xi^{j}\left(P_{m}\left(\kappa_{0}, \kappa_{1}, \vee\right)\right)$ for each $\vee>0$ implies that $\left\{\kappa_{m}\right\}$ is a Cauchy $E$-sequence in $\left(H, E, P_{m}\right)$. 
The E-completeness of $\left(H, E, P_{m}\right)$ leads to the existence of a point $\kappa_{*} \in H$ with $\lim _{m \rightarrow \infty} P_{m}\left(\kappa_{m}, \kappa_{*}, \curlyvee\right)=0$ for all $\curlyvee>0$ and $\kappa_{m} \sim \kappa_{*}$ for each $m \geq k$, for some $k$. By (d), we also conclude that $\alpha\left(\kappa_{m}, \kappa_{*}\right) \geq 1$ for each $m \geq k$. Now, from (3), we get

$$
\begin{aligned}
P_{m}\left(T \kappa_{m}, T \kappa_{*}, \curlyvee\right) & \leq \alpha\left(\kappa_{m}, \kappa_{*}\right) P_{m}\left(T \kappa_{m}, T \kappa_{*}, \curlyvee\right) \\
& \leq Q\left(P_{m}\left(\kappa_{m}, \kappa_{*}, \curlyvee\right), P_{m}\left(\kappa_{m}, T \kappa_{m}, \curlyvee\right), P_{m}\left(\kappa_{*}, T \kappa_{*}, \curlyvee\right), \frac{1}{2}\left(P_{m}\left(\kappa_{*}, T \kappa_{m}, \curlyvee\right)+P_{m}\left(\kappa_{m}, T \kappa_{*}, \curlyvee\right)\right)\right) \\
& =Q\left(P_{m}\left(\kappa_{m}, \kappa_{*}, \curlyvee\right), P_{m}\left(\kappa_{m}, \kappa_{m+1}, \curlyvee\right), P_{m}\left(\kappa_{*}, T \kappa_{*}, \curlyvee\right), \frac{1}{2}\left(P_{m}\left(\kappa_{m+1}, \kappa_{*}, \curlyvee\right)+P_{m}\left(\kappa_{m}, T \kappa_{*}, \curlyvee\right)\right)\right),
\end{aligned}
$$

for all $\curlyvee>0$.

By triangle property and (12), we get

$$
\begin{aligned}
& P_{m}\left(\kappa_{*}, T \kappa_{*}, \curlyvee\right) \leq P_{m}\left(\kappa_{*}, \kappa_{m+1}, \curlyvee\right)+P_{m}\left(\kappa_{m+1}, T \kappa_{*}, \curlyvee\right)=P_{m}\left(\kappa_{*}, \kappa_{m+1}, \curlyvee\right)+P_{m}\left(T \kappa_{m}, T \kappa_{*}, \curlyvee\right) \\
& \leq P_{m}\left(\kappa_{*}, \kappa_{m+1}, \curlyvee\right)+Q\left(P_{m}\left(\kappa_{m}, \kappa_{*}, \curlyvee\right), P_{m}\left(\kappa_{m}, \kappa_{m+1}, \curlyvee\right), P_{m}\left(\kappa_{*}, T \kappa_{*}, \curlyvee\right), \frac{1}{2}\left(P_{m}\left(\kappa_{m+1}, \kappa_{*}, \curlyvee\right)+P_{m}\left(\kappa_{m}, T \kappa_{*}, \curlyvee\right)\right)\right) \\
& \leq P_{m}\left(\kappa_{*}, \kappa_{m+1}, \curlyvee\right)+Q\left(P_{m}\left(\kappa_{m}, \kappa_{*}, \curlyvee\right), P_{m}\left(\kappa_{m}, \kappa_{m+1}, \curlyvee\right), P_{m}\left(\kappa_{*}, T \kappa_{*}, \curlyvee\right), \frac{1}{2}\left(P_{m}\left(\kappa_{m+1}, \kappa_{*}, \curlyvee\right)+P_{m}\left(\kappa_{m}, \kappa_{*}, \curlyvee\right)+P_{m}\left(\kappa_{*}, T \kappa_{*}, \curlyvee\right)\right)\right) \text {, }
\end{aligned}
$$

for all $r>0$.

Applying the limit when $m \longrightarrow \infty$ in (13), we get

$$
P_{m}\left(\kappa_{*}, T \kappa_{*}, \curlyvee\right) \leq Q\left(0,0, P_{m}\left(\kappa_{*}, T \kappa_{*}, \curlyvee\right), \frac{1}{2}\left(P_{m}\left(\kappa_{*}, T \kappa_{*}, \curlyvee\right)\right)\right),
$$

for all $\curlyvee>0$.
Thus, by property (iv) of $Q_{\xi}$, we get $P_{m}\left(\kappa_{*}, T \kappa_{*}, \curlyvee\right)=0$ for all $\vee>0$. Therefore, $\kappa_{*}=T \kappa_{*}$.

In the following result, (15) is a special case of (3), but with (15) we do not need the condition of the regularity of the $E$-parametric metric space.

Theorem 5. Let $\left(H, E, P_{m}\right)$ be an E-complete E-parametric metric space. Let $T: H \longrightarrow H$ and $\alpha: H \times H \longrightarrow[0, \infty)$ be two maps such that

$$
\alpha\left(\ell, \ell^{\prime}\right) P_{m}\left(T \ell, T \ell^{\prime}, \vee\right) \leq \xi\left(\max \left\{P_{m}\left(\ell, \ell^{\prime}, \curlyvee\right), P_{m}(\ell, T \ell, \vee), P_{m}\left(\ell^{\prime}, T \ell^{\prime}, \vee\right), \frac{P_{m}\left(\ell^{\prime}, T \ell, \vee\right)+P_{m}\left(\ell, T \ell^{\prime}, \vee\right)}{2}\right\}\right),
$$

for all $\vee>0$ and for all $\ell, \ell^{\prime} \in H$ with $\ell \sim \ell^{\prime}$, where $\xi: \mathbb{R}^{+}$ $\longrightarrow \mathbb{R}^{+}$is a continuous and nondecreasing map with $\sum_{n=1}^{\infty}$ $\xi^{n}(t)<\infty$ for all $t \geq 0$ and $\xi(t)<t$ for all $t>0$. Also, consider the following assertions:

(a) $T$ is E-preserving, that is, $\ell \sim \ell^{\prime}$ implies $T \ell \sim T \ell^{\prime}$

(b) There is $\ell_{0} \in H$ such that $\ell_{0} \sim T \ell_{0}$ and $\alpha\left(\ell_{0}, T \ell_{0}\right) \geq 1$

(c) For every $\ell, \ell^{\prime} \in H$ such that $\ell \sim \ell^{\prime}$ and $\alpha\left(\ell, \ell^{\prime}\right) \geq 1$, we have $\alpha\left(T \ell, T \ell^{\prime}\right) \geq 1$ (d) For each E-sequence $\left\{\ell_{m}\right\}$ in $H$ with $\alpha\left(\ell_{m}, \ell_{m+1}\right) \geq 1$ for all $m \in \mathbb{N}$ and $\ell_{m} \longrightarrow \ell$, we have $\alpha\left(\ell_{m}, \ell\right) \geq 1$ for all $m \in \mathbb{N}$

Then, $T$ has a fixed point.

Proof. By (b), we know that there is $\kappa_{0} \in H$ of the form $\kappa_{0} \sim T \kappa_{0}$ and $\alpha\left(\kappa_{0}, T \kappa_{0}\right) \geq 1$. In view of the fact that $T$ has E-preserving nature, thus $T \kappa_{0} \sim T^{2} \kappa_{0}$ and by (c), $\alpha(T$ $\left.\kappa_{0}, T^{2} \kappa_{0}\right) \geq 1$. Continuing this process, we get $T^{m} \kappa_{0} \sim$ 
$T^{m+1} \kappa_{0}$ and $\alpha\left(T^{m} \kappa_{0}, T^{m+1} \kappa_{0}\right) \geq 1$ for all $m \in \mathbb{N}$. Take a sequence $\left\{\kappa_{m}\right\}$ with the terms as $\kappa_{m}=T^{m} \kappa_{0}$ for all $m \in$ $\mathbb{N}$. Thus, $\kappa_{m} \sim \kappa_{m+1}$ and $\alpha\left(\kappa_{m}, \kappa_{m+1}\right) \geq 1$ for all $m \in \mathbb{W}$.
Now, we take $\kappa_{m} \neq \kappa_{m+1}$ for all $m \in \mathbb{W}$. From (15), one writes for each $m \in \mathbb{W}$,

$$
\begin{aligned}
P_{m}\left(\kappa_{m+1}, \kappa_{m+2}, \vee\right) & =P_{m}\left(T \kappa_{m}, T \kappa_{m+1}, \vee\right) \leq \alpha\left(\kappa_{m}, \kappa_{m+1}\right) P_{m}\left(T \kappa_{m}, T \kappa_{m+1}, \vee\right) \\
& \leq \xi\left(\max \left\{P_{m}\left(\kappa_{m}, \kappa_{m+1}, \vee\right), P_{m}\left(\kappa_{m}, T \kappa_{m}, \vee\right), P_{m}\left(\kappa_{m+1}, T \kappa_{m+1}, \vee\right), \frac{1}{2}\left(P_{m}\left(\kappa_{m+1}, T \kappa_{m}, \vee\right)+P_{m}\left(\kappa_{m}, T \kappa_{m+1}, \vee\right)\right)\right\}\right. \\
& =\xi\left(\max \left\{P_{m}\left(\kappa_{m}, \kappa_{m+1}, \vee\right), P_{m}\left(\kappa_{m}, \kappa_{m+1}, \vee\right), P_{m}\left(\kappa_{m+1}, \kappa_{m+2}, \vee\right), \frac{1}{2}\left(P_{m}\left(\kappa_{m+1}, \kappa_{m+1}, \vee\right)+P_{m}\left(\kappa_{m}, \kappa_{m+2}, \vee\right)\right)\right\}\right) \\
& \leq \xi\left(\max \left\{P_{m}\left(\kappa_{m}, \kappa_{m+1}, \vee\right), P_{m}\left(\kappa_{m+1}, \kappa_{m+2}, \vee\right)\right\}\right),
\end{aligned}
$$

for all $\curlyvee>0$.

Since $\kappa_{m} \neq \kappa_{m+1}$ for all $m \in \mathbb{W}$, (16) implies that $P_{m}\left(\kappa_{m+1}, \kappa_{m+2}, \vee\right) \leq \xi\left(P_{m}\left(\kappa_{m}, \kappa_{m+1}, \vee\right)\right)$, for all $\vee>0$ and $m \in \mathbb{W}$.

Therefore,

$P_{m}\left(\kappa_{m}, \kappa_{m+1}, \vee\right) \leq \xi^{m}\left(P_{m}\left(\kappa_{0}, \kappa_{1}, \vee\right)\right)$, for all $\vee>0$ and $m \in \mathbb{N}$

$$
\begin{aligned}
P_{m}\left(T \kappa_{m}, T \kappa_{*}, \vee\right) & \leq \alpha\left(\kappa_{m}, \kappa_{*}\right) P_{m}\left(T \kappa_{m}, T \kappa_{*}, \vee\right) \\
& \leq \xi\left(\max \left\{P_{m}\left(\kappa_{m}, \kappa_{*}, \vee\right), P_{m}\left(\kappa_{m}, T \kappa_{m}, \vee\right), P_{m}\left(\kappa_{*}, T \kappa_{*}, \vee\right), \frac{1}{2}\left(P_{\mathrm{m}}\left(\kappa_{*}, T \kappa_{m}, \vee\right)+P_{m}\left(\kappa_{m}, T \kappa_{*}, \vee\right)\right)\right\}\right) \\
& =\xi\left(\max \left\{P_{m}\left(\kappa_{m}, \kappa_{*}, \vee\right), P_{m}\left(\kappa_{m}, \kappa_{m+1}, \vee\right), P_{m}\left(\kappa_{*}, T \kappa_{*}, \vee\right), \frac{1}{2}\left(P_{m}\left(\kappa_{m+1}, \kappa_{*}, \vee\right)+P_{m}\left(\kappa_{m}, T \kappa_{*}, \vee\right)\right)\right\}\right),
\end{aligned}
$$

for all $\vee>0$ and for all $m \geq k$.

By triangle property and (19), we get

$$
\begin{aligned}
& P_{m}\left(\kappa_{*}, T \kappa_{*}, \curlyvee\right) \leq P_{m}\left(\kappa_{*}, \kappa_{m+1}, \curlyvee\right)+P_{m}\left(\kappa_{m+1}, T \kappa_{*}, \curlyvee\right)=P_{m}\left(\kappa_{*}, \kappa_{m+1}, \curlyvee\right)+P_{m}\left(T \kappa_{m}, T \kappa_{*}, \curlyvee\right) \\
& \leq P_{m}\left(\kappa_{*}, \kappa_{m+1}, \vee\right)+\xi\left(\max \left\{P_{m}\left(\kappa_{m}, \kappa_{*}, \curlyvee\right), P_{m}\left(\kappa_{m}, \kappa_{m+1}, \curlyvee\right), P_{m}\left(\kappa_{*}, T \kappa_{*}, \curlyvee\right), \frac{1}{2}\left(P_{m}\left(\kappa_{m+1}, \kappa_{*}, \curlyvee\right)+P_{m}\left(\kappa_{m}, T \kappa_{*}, \curlyvee\right)\right)\right\}\right) \\
& \leq P_{m}\left(\kappa_{*}, \kappa_{m+1}, \curlyvee\right)+\xi\left(\max \left\{P_{m}\left(\kappa_{m}, \kappa_{*}, \curlyvee\right), P_{m}\left(\kappa_{m}, \kappa_{m+1}, \curlyvee\right), P_{m}\left(\kappa_{*}, T \kappa_{*}, \curlyvee\right), \frac{1}{2}\left(P_{m}\left(\kappa_{m+1}, \kappa_{*}, \curlyvee\right)+P_{m}\left(\kappa_{m}, \kappa_{*}, \vee\right)+P_{m}\left(\kappa_{*}, T \kappa_{*}, \curlyvee\right)\right)\right\}\right),
\end{aligned}
$$


for all $\vee>0$ and for all $m \geq k$.

Applying the limit when $m \longrightarrow \infty$ in (20) and using the continuity of $\xi$, we get

$$
P_{m}\left(\kappa_{*}, T \kappa_{*}, \curlyvee\right) \leq \xi\left(\max \left\{P_{m}\left(\kappa_{*}, T \kappa_{*}, \curlyvee\right), \frac{1}{2}\left(P_{m}\left(\kappa_{*}, T \kappa_{*}, \curlyvee\right)\right)\right\}\right),
$$

for all $\vee>0$.

The above inequality is only possible when $P_{m}\left(\kappa_{*}, T \kappa_{*}\right.$, $\checkmark)=0$ for all $\vee>0$. Therefore, $\kappa_{*}=T \kappa_{*}$.

Example 6. Take $H=C[1, \infty)$ with equivalence relation $E$ on $H$ defined as $h_{g} \sim h_{f}$ if $t \mid h_{g}(t)-h_{f}(t)$. Define $P_{m}: H \times H$ $\times(0, \infty) \longrightarrow[0, \infty)$ by

$P_{m}\left(h_{g}, h_{f}, \curlyvee\right)=\left\{\begin{array}{l}\left|h_{g}(\vee)-h_{f}(\vee)\right|, \text { for all } h_{g}, h_{f} \in H \text { and for all } \vee \geq 1, \\ 0, \text { for all } h_{g}, h_{f} \in H \text { and for all } \vee \in(0,1) .\end{array}\right.$

Define the maps $T: H \longrightarrow H$ and $\alpha: H \times H \longrightarrow[0, \infty)$ by

$$
\begin{aligned}
& T\left(h_{g}(t)\right)= \begin{cases}\frac{h_{g}(t)+1}{2}, & \text { if } h_{g}(t) \geq 0, \\
-\left(h_{g}(t)\right)^{2}, & \text { otherwise, }\end{cases} \\
& \alpha\left(h_{g}, h_{f}\right)= \begin{cases}1, & \text { if } h_{g}, h_{f} \geq 0, \\
0, & \text { otherwise. }\end{cases}
\end{aligned}
$$

Here, one can verify that all the axioms of Theorem 5 are valid, and there is a fixed point of $T$ in $H$.
Let $\left(H, E, P_{m}\right)$ be an $E$-parametric metric space. Let $\mathcal{N}(H, E)$ denotes the collection of all nonempty subsets of $H$ having the following properties:

(i) For any $A_{H} \in \mathcal{N}(H, E)$, sup $\left\{P_{m}(\kappa, \omega, \curlyvee): \kappa, \omega \in A_{H}\right\}$ exists for each $\vee>0$

(ii) If $\left\{\kappa_{n}\right\} \subset A_{H}$ and $\kappa_{n} \longrightarrow \kappa$, then $\kappa \in A_{H}$

The Hausdroff $E$-parametric distance $H_{P_{m}}$ is a mapping from $\mathcal{N}(H, E) \times \mathcal{N}(H, E) \times(0, \infty)$ into $[0, \infty)$ defined by

$$
H_{P_{m}}\left(A_{H}, B_{H}, \curlyvee\right)=\max \left\{\lambda\left(A_{H}, B_{H}, \curlyvee\right), \lambda\left(B_{H}, A_{H}, \curlyvee\right)\right\},
$$

for all $\vee>0$ where

$$
\begin{aligned}
& \lambda\left(A_{H}, B_{H}, \vee\right)=\sup \left\{\inf \left\{P_{m}(\kappa, \omega, \vee): \omega \in B_{H}\right\}: \kappa \in A_{H}\right\} \\
& \lambda\left(B_{H}, A_{H}, \curlyvee\right)=\sup \left\{\inf \left\{P_{m}(\omega, \kappa, \vee): \kappa \in A_{H}\right\}: \omega \in B_{H}\right\} .
\end{aligned}
$$

Here, everyone should know that for each $A_{H} \in \mathcal{N}(H, E)$, $\omega \in\left(H, E, P_{m}\right)$ and for each value of $\checkmark$ there must exists $\kappa_{q}$ $\in A_{H}$ with $P_{m}\left(\kappa_{q}, \omega, \vee\right) \leq q \inf \left\{P_{m}(\kappa, \omega, \curlyvee): \kappa \in A_{H}\right\}$, where $q>1$. Subsequently, we take inf $\left\{P_{m}(\omega, \kappa, \vee): \kappa \in A_{H}\right\}=P_{m}$ $\left(\omega, A_{H}, \curlyvee\right)$.

In the following theorem, we will discuss the case of the existence of fixed points for multivalued maps.

Theorem 7. Let $\left(H, E, P_{m}\right)$ be an E-complete regular $E$ -parametric metric space. Let $T: \mathcal{N}(H, E) \longrightarrow \mathcal{N}(H, E)$ and $\alpha: H \times H \longrightarrow[0, \infty)$ be two maps such that

$$
\alpha_{c}\left(T \ell, T \ell^{\prime}\right) H_{P_{m}}\left(T \ell, T \ell^{\prime}, \vee\right) \leq Q\left(P_{m}\left(\ell, \ell^{\prime}, \vee\right), P_{m}(\ell, T \ell, \vee), P_{m}\left(\ell^{\prime}, T \ell^{\prime}, \vee\right), \frac{P_{m}\left(\ell^{\prime}, T \ell, \vee\right)+P_{m}\left(\ell, T \ell^{\prime}, \vee\right)}{2}\right),
$$

for all $\vee>0$ and for all $\ell, \ell^{\prime} \in H$ with $\ell \sim \ell^{\prime}$, where $Q \in Q_{\xi}$ and $\alpha_{c}\left(T \ell, T \ell^{\prime}\right)=\inf \left\{\alpha\left(j, j^{\prime}\right): j \in T \ell, j^{\prime} \in T \ell^{\prime}\right\}$. Also, consider the following assertions:

(a) $T$ is E-preserving, that is, $\ell \sim \ell^{\prime}$ implies $j \sim j^{\prime}$ for each $j \in T \ell$ and $j^{\prime} \in T \ell^{\prime}$

(b) There is $\kappa_{0} \in H$ and $\kappa_{1} \in T \kappa_{0}$ such that $\kappa_{0} \sim \kappa_{1}$ and $\alpha\left(\kappa_{0}, \kappa_{1}\right)>1$

(c) For every $\ell, \ell^{\prime} \in H$ such that $\ell \sim \ell^{\prime}$ and $\alpha\left(\ell, \ell^{\prime}\right)>1$, we have $\alpha_{c}\left(T \ell, T \ell^{\prime}\right)>1$ (d) For each E-sequence $\left\{\ell_{m}\right\}$ in $H$ with $\alpha\left(\ell_{m}, \ell_{m+1}\right)>1$ for all $m \in \mathbb{N}$ and $\ell_{m} \longrightarrow \ell$, we have $\alpha\left(\ell_{m}, \ell\right)>1$ for all $m \in \mathbb{N}$

(e) For each $q>1, T \kappa \in \mathcal{N}(H, E)$ and $\omega \in H$ there is $\kappa_{q}$ $\in T \kappa$ with $P_{m}\left(\kappa_{q}, \omega, \curlyvee\right) \leq q P_{m}(\omega, T \kappa, \curlyvee)$ for all $\curlyvee>0$, where inf $\left\{P_{m}\left(\omega, \kappa_{*}, \curlyvee\right): \kappa_{*} \in T \kappa\right\}=P_{m}(\omega, T \kappa, \vee)$.

Then, $T$ has a fixed point.

Proof. By (b) and (c), we observe that $\alpha_{c}\left(T \kappa_{0}, T \kappa_{1}\right)>1$, since $\kappa_{0} \in H$ and $\kappa_{1} \in T \kappa_{0}$ are of the form $\kappa_{0} \sim \kappa_{1}$ and $\alpha\left(\kappa_{0}, \kappa_{1}\right)>1$. 
Here, we take $\kappa_{0} \neq \kappa_{1}$. Otherwise, $\kappa_{0} \in T \kappa_{0}$. By (26), we get

$$
\begin{aligned}
\alpha_{c}\left(T \kappa_{0}, T \kappa_{1}\right) P_{m}\left(\kappa_{1}, T \kappa_{1}, \vee\right) & \leq \alpha_{c}\left(T \kappa_{0}, T \kappa_{1}\right) H_{P_{m}}\left(T \kappa_{0}, T \kappa_{1}, \vee\right) \\
& \leq Q\left(P_{m}\left(\kappa_{0}, \kappa_{1}, \vee\right), P_{m}\left(\kappa_{0}, T \kappa_{0}, \curlyvee\right), P_{m}\left(\kappa_{1}, T \kappa_{1}, \vee\right), \frac{1}{2}\left(P_{m}\left(\kappa_{1}, T \kappa_{0}, \curlyvee\right)+P_{m}\left(\kappa_{0}, T \kappa_{1}, \vee\right)\right)\right)
\end{aligned}
$$

for all $\vee>0$.

Since $\alpha_{c}\left(T \kappa_{0}, T \kappa_{1}\right)>1$, by (e), there is $\kappa_{2} \in T \kappa_{1}$ such that

$$
P_{m}\left(\kappa_{1}, \kappa_{2}, \vee\right) \leq \alpha_{c}\left(T \kappa_{0}, T \kappa_{1}\right) P_{m}\left(\kappa_{1}, T \kappa_{1}, \vee\right)
$$

$$
\begin{aligned}
& P_{m}\left(\kappa_{1}, \kappa_{2}, \vee\right) \leq \alpha_{c}\left(T \kappa_{0}, T \kappa_{1}\right) P_{m}\left(\kappa_{1}, T \kappa_{1}, \vee\right) \leq Q\left(P_{m}\left(\kappa_{0}, \kappa_{1}, \vee\right), P_{m}\left(\kappa_{0}, T \kappa_{0}, \vee\right), P_{m}\left(\kappa_{1}, T \kappa_{1}, \vee\right), \frac{1}{2}\left(P_{m}\left(\kappa_{1}, T \kappa_{0}, \vee\right)+P_{m}\left(\kappa_{0}, T \kappa_{1}, \vee\right)\right)\right) \\
& \leq Q\left(P_{m}\left(\kappa_{0}, \kappa_{1}, \vee\right), P_{m}\left(\kappa_{0}, \kappa_{1}, \vee\right), P_{m}\left(\kappa_{1}, \kappa_{2}, \vee\right), \frac{1}{2}\left(P_{m}\left(\kappa_{0}, \kappa_{1}, \vee\right)+P_{m}\left(\kappa_{1}, \kappa_{2}, \vee\right)\right)\right),
\end{aligned}
$$

for all $\vee>0$.

Here, our claim is $P_{m}\left(\kappa_{0}, \kappa_{1}, \curlyvee\right)>P_{m}\left(\kappa_{1}, \kappa_{2}, \curlyvee\right) \forall \curlyvee>0$. Suppose this is not true. Then, $P_{m}\left(\kappa_{0}, \kappa_{1}, \vee^{0}\right) \leq P_{m}\left(\kappa_{1}, \kappa_{2}\right.$, $\vee^{0}$ ) for some $\vee^{0}>0$. Then, from (29) and by property (i) of $Q_{\xi}$, for $\vee^{0}>0$, we get

$$
\begin{aligned}
& P_{m}\left(\kappa_{1}, \kappa_{2}, \vee^{0}\right) \leq Q\left(P_{m}\left(\kappa_{1}, \kappa_{2}, \vee^{0}\right), P_{m}\left(\kappa_{0}, \kappa_{1}, \vee^{0}\right), P_{m}\right. \\
& \left.\cdot\left(\kappa_{1}, \kappa_{2}, \vee^{0}\right), P_{m}\left(\kappa_{1}, \kappa_{2}, \vee^{0}\right)\right) .
\end{aligned}
$$

From property (iii) of $Q_{\xi}$ and the above inequality, we get

$$
P_{m}\left(\kappa_{1}, \kappa_{2}, \vee^{0}\right)=0 \text {, for some } \vee^{0}>0
$$

This fact together with regularity condition implies

$$
P_{m}\left(\kappa_{1}, \kappa_{2}, \curlyvee\right)=0 \text {, for all } \vee>0,
$$

and it is not possible under the assumption. Thus, $P_{m}\left(\kappa_{0}\right.$, $\left.\kappa_{1}, \vee\right)>P_{m}\left(\kappa_{1}, \kappa_{2}, \curlyvee\right)$ for all $\vee>0$. Again, we use (29) and property (i) of $Q_{\xi}$ to obtain

$$
\begin{aligned}
& P_{m}\left(\kappa_{1}, \kappa_{2}, \curlyvee\right) \leq Q\left(P_{m}\left(\kappa_{0}, \kappa_{1}, \curlyvee\right), P_{m}\left(\kappa_{0}, \kappa_{1}, \curlyvee\right), P_{m}\right. \\
& \left.\cdot\left(\kappa_{1}, \kappa_{2}, \curlyvee\right), P_{m}\left(\kappa_{0}, \kappa_{1}, \curlyvee\right)\right), \text { for all } \vee>0 .
\end{aligned}
$$

The property (ii) of $Q_{\xi}$ and (33) imply

$$
P_{m}\left(\kappa_{1}, \kappa_{2}, \curlyvee\right) \leq \xi\left(P_{m}\left(\kappa_{0}, \kappa_{1}, \curlyvee\right)\right) \text {, for all } \vee>0
$$

for all $\vee>0$. Here, again, we take $\kappa_{1} \neq \kappa_{2}$. Otherwise, $\kappa_{1} \in T \kappa_{1}$. Thus, by using it in (27), we get
Clearly, $\alpha\left(\kappa_{1}, \kappa_{2}\right)>1$ due to the fact that $\kappa_{1} \in T \kappa_{0}, \kappa_{2} \epsilon$ $T \kappa_{1}$ and $\alpha_{c}\left(T \kappa_{0}, T \kappa_{1}\right)>1$. Also, the E-preserving characteristic of $T$ yields $\kappa_{1} \sim \kappa_{2}$. Thus, by (c), we get $\alpha_{c}\left(T \kappa_{1}, T \kappa_{2}\right)$ $>1$. The repeated application of the above steps yields the sequence $\left\{\kappa_{m}\right\}$ with $\kappa_{m} \in T \kappa_{m-1}, \kappa_{m-1} \sim \kappa_{m}, \alpha\left(\kappa_{m-1}, \kappa_{m}\right)>1$ and $P_{m}\left(\kappa_{m-1}, \kappa_{m}, \curlyvee\right)>P_{m}\left(\kappa_{m}, \kappa_{m+1}, \curlyvee\right)$ for all $\curlyvee>0$ and

$$
P_{m}\left(\kappa_{m}, \kappa_{m+1}, \vee\right) \leq \xi^{m}\left(P_{m}\left(\kappa_{0}, \kappa_{1}, \vee\right)\right)
$$

for all $\vee>0$ and for all $m \in \mathbb{N}$.

The steps given below will show that $\left\{\kappa_{m}\right\}$ is a Cauchy $E$ -sequence. For each $n>m \in \mathbb{N}$, the triangle inequality and (35) imply that

$$
P_{m}\left(\kappa_{m}, \kappa_{n}, \curlyvee\right) \leq \sum_{j=m}^{n-1} P_{m}\left(\kappa_{j}, \kappa_{j+1}, \vee\right) \leq \sum_{j=m}^{\infty} \xi^{j}\left(P_{m}\left(\kappa_{0}, \kappa_{1}, \vee\right)\right)
$$

for all $\vee>0$.

The convergence of $\sum_{j=1}^{\infty} \xi^{j}\left(P_{m}\left(\kappa_{0}, \kappa_{1}, \vee\right)\right)$ together with the above inequality confirm that $\left\{\kappa_{m}\right\}$ is a Cauchy $E$ -sequence in $\left(H, E, P_{m}\right)$. The $E$-completeness of $\left(H, E, P_{m}\right)$ ensures the existence of a point $\kappa_{*} \in H$ with $\lim _{m \rightarrow \infty} P_{m}($ $\left.\kappa_{m}, \kappa_{*}, \curlyvee\right)=0$ for all $\vee>0$ and $\kappa_{m} \sim \kappa_{*}$ for each $m \geq \mathrm{k}$, for some $k$. We now able to conclude that, by (d), $\alpha\left(\kappa_{m}, \kappa_{*}\right)$ $>1$ for each $m \in \mathbb{N}$. Since $\kappa_{m} \sim \kappa_{*}$ and $\alpha\left(\kappa_{m}, \kappa_{*}\right)>1$ for each $m \geq k$, using (c), we have $\alpha\left(T \kappa_{m}, T \kappa_{*}\right)>1$. Thus, by (26), we get 


$$
\begin{aligned}
H_{P_{m}}\left(T \kappa_{m}, T \kappa_{*}, \curlyvee\right) & <\alpha\left(T \kappa_{m}, T \kappa_{*}\right) H_{P_{m}}\left(T \kappa_{m}, T \kappa_{*}, \curlyvee\right) \\
& \leq Q\left(P_{m}\left(\kappa_{m}, \kappa_{*}, \curlyvee\right), P_{m}\left(\kappa_{m}, T \kappa_{m}, \curlyvee\right), P_{m}\left(\kappa_{*}, T \kappa_{*}, \curlyvee\right), \frac{1}{2}\left(P_{m}\left(\kappa_{*}, T \kappa_{m}, \curlyvee\right)+P_{m}\left(\kappa_{m}, T \kappa_{*}, \curlyvee\right)\right)\right) \\
& =Q\left(P_{m}\left(\kappa_{m}, \kappa_{*}, \curlyvee\right), P_{m}\left(\kappa_{m}, \kappa_{m+1}, \curlyvee\right), P_{m}\left(\kappa_{*}, T \kappa_{*}, \curlyvee\right), \frac{1}{2}\left(P_{m}\left(\kappa_{m+1}, \kappa_{*}, \curlyvee\right)+P_{m}\left(\kappa_{m}, T \kappa_{*}, \curlyvee\right)\right)\right),
\end{aligned}
$$

for all $\vee>0$.

By triangle property and (37), we get

$$
\begin{aligned}
& P_{m}\left(\kappa_{*}, T \kappa_{*}, \curlyvee\right) \leq P_{m}\left(\kappa_{*}, \kappa_{m+1}, \curlyvee\right)+P_{m}\left(\kappa_{m+1}, T \kappa_{*}, \curlyvee\right) \leq P_{m}\left(\kappa_{*}, \kappa_{m+1}, \curlyvee\right)+H_{P_{m}}\left(T \kappa_{m}, T \kappa_{*}, \curlyvee\right)<P_{m}\left(\kappa_{*}, \kappa_{m+1}, \curlyvee\right) \\
& +Q\left(P_{m}\left(\kappa_{m}, \kappa_{*}, \curlyvee\right), P_{m}\left(\kappa_{m}, \kappa_{m+1}, \curlyvee\right), P_{m}\left(\kappa_{*}, T \kappa_{*}, \curlyvee\right), \frac{1}{2}\left(P_{m}\left(\kappa_{m+1}, \kappa_{*}, \curlyvee\right)+P_{m}\left(\kappa_{m}, T \kappa_{*}, \curlyvee\right)\right)\right) \\
& \leq P_{m}\left(\kappa_{*}, \kappa_{m+1}, \curlyvee\right)+Q\left(P_{m}\left(\kappa_{m}, \kappa_{*}, \curlyvee\right), P_{m}\left(\kappa_{m}, \kappa_{m+1}, \curlyvee\right), P_{m}\left(\kappa_{*}, T \kappa_{*}, \curlyvee\right), \frac{1}{2}\left(P_{m}\left(\kappa_{m+1}, \kappa_{*}, \curlyvee\right)+P_{m}\left(\kappa_{m}, \kappa_{*}, \curlyvee\right)+P_{m}\left(\kappa_{*}, T \kappa_{*}, \curlyvee\right)\right)\right),
\end{aligned}
$$

for all $\vee>0$.

Applying the limit when $m \longrightarrow \infty$ in (38), we get $P_{m}\left(\kappa_{*}, T \kappa_{*}, \curlyvee\right) \leq Q\left(0,0, P_{m}\left(\kappa_{*}, T \kappa_{*}, \curlyvee\right), \frac{1}{2}\left(P_{m}\left(\kappa_{*}, T \kappa_{*}, \curlyvee\right)\right)\right)$, for all $\curlyvee>0$.

Thus, by property (iv) of $Q_{\xi}$, we get $P_{m}\left(\kappa_{*}, T \kappa_{*}, \curlyvee\right)=0$ for all $\vee>0$. Therefore, $\kappa_{*} \in T \kappa_{*}$.
In the following theorem, (40) is a special case of (26), but with (40), we do not need the condition of regularity of the $E$-parametric metric space.

Theorem 8. Let $\left(H, E, P_{m}\right)$ be an E-complete E-parametric metric space. Let $T: \mathcal{N}(H, E) \longrightarrow \mathcal{N}(H, E)$ and $\alpha: H \times H$ $\longrightarrow[0, \infty)$ be two maps such that

$$
\alpha_{c}\left(T \ell, T \ell^{\prime}\right) H_{P_{m}}\left(T \ell, T \ell^{\prime}, \vee\right) \leq \xi\left(\max \left\{P_{m}\left(\ell, \ell^{\prime}, \vee\right), P_{m}(\ell, T \ell, \vee), P_{m}\left(\ell^{\prime}, T \ell^{\prime}, \vee\right), \frac{P_{m}\left(\ell^{\prime}, T \ell, \vee\right)+P_{m}\left(\ell, T \ell^{\prime}, \vee\right)}{2}\right\}\right),
$$

for all $\vee>0$ and for all $\ell, \ell^{\prime} \in H$ with $\ell \sim \ell^{\prime}$, where $\xi: \mathbb{R}^{+}$ $\longrightarrow \mathbb{R}^{+}$is a continuous and nondecreasing mapping with $\sum_{n=1}^{\infty} \xi^{n}(t)<\infty$ for all $t \geq 0$ and $\xi(t)<t$ for all $t>0$, and $\alpha_{c}$ $\left(T \ell, T \ell^{\prime}\right)=\inf \left\{\alpha\left(j, j^{\prime}\right): j \in T \ell, j^{\prime} \in T \ell^{\prime}\right\}$. Also, consider the following assertions:

(a) $T$ is E-preserving, that is, $\ell \sim \ell^{\prime}$ implies $j \sim j^{\prime}$ for each $j \in T \ell$ and $j^{\prime} \in T \ell^{\prime}$

(b) There is $\kappa_{0} \in H$ and $\kappa_{1} \in T \kappa_{0}$ such that $\kappa_{0} \sim \kappa_{1}$ and $\alpha\left(\kappa_{0}, \kappa_{1}\right)>1$ (c) For every $\ell, \ell^{\prime} \in H$ such that $\ell \sim \ell^{\prime}$ and $\alpha\left(\ell, \ell^{\prime}\right)>1$, we have $\alpha_{c}\left(T \ell, T \ell^{\prime}\right)>1$

(d) For each E-sequence $\left\{\ell_{m}\right\}$ in $H$ with $\alpha\left(\ell_{m}, \ell_{m+1}\right)>1$ for all $m \in \mathbb{N}$ and $\ell_{m} \longrightarrow \ell$, we have $\alpha\left(\ell_{m}, \ell\right)>1$

(e) For each $q>1, \quad T \kappa \in \mathcal{N}(H, E)$ and $\omega \in H$ there exists $\kappa_{q} \in T \kappa$ with $P_{m}\left(\kappa_{q}, \omega, \curlyvee\right) \leq q P_{m}(\omega, T \kappa, \curlyvee)$ for all $\vee>0$, where $\inf \left\{P_{m}\left(\omega, \kappa_{*}, \curlyvee\right): \kappa_{*} \in T \kappa\right\}=P_{m}(\omega$, $T \kappa, \curlyvee)$.

Then, $T$ has a fixed point. 
The proof of this theorem will be done by following the techniques of Theorems 5 and 6.

\section{Application to Existence of Solutions of Integral Equations}

Let $X=C([0, T], \mathbb{R})$ be the set of real continuous functions defined on $[0, T]$ and $P_{m}: X \times X \times(0, \infty) \longrightarrow[0,+\infty)$ be defined by

$$
P_{m}(x, y, \alpha)=\sup _{t \in[0, T]} \frac{1}{1+\left(\alpha t^{2}\right)}|x(t)-y(t)|,
$$

for all $x, y \in X$ and all $t \in[0, T]$. Then, $\left(X, P_{m}\right)$ is a complete parametric metric space. Let $\sim$ be the equivalence relation on $X$ defined by $x \sim y$ if and only if $t \mid x(t)-y(t)$. Then, $(X$, $\left.P_{m}, \sim\right)$ is a complete $E$-parametric metric space. Consider the following integral equation:

$$
x(t)=p(t)+\int_{0}^{T} S(t, s) f(s, x(s)) d s
$$

where
(A) $f:[0, T] \times \mathbb{R} \longrightarrow \mathbb{R}$ is continuous
(B) $p:[0, T] \longrightarrow \mathbb{R}$ is continuous
(C) $S:[0, T] \times[0, T] \longrightarrow[0,+\infty)$ is continuous and

$$
\sup _{t \in[0, T]}\left(\int_{0}^{T} S(t, s) d s\right) \leq 1
$$

(D) There exists $k \in(0,1)$ such that

$$
0 \leq[f(s, y(s))-f(s, \mathrm{x}(s))] \leq \frac{k}{1+\left(\alpha s^{2}\right)} \max \left\{|x(s)-y(s)|,|x(s)-H x(s)|,|y(s)-H y(s)|, \frac{|x(s)-H y(s)|+|y(s)-H x(s)|}{2}\right\}
$$

for all $x, y \in X$ with $x \sim y$ and $s \in[0, T]$ where

$$
H x(t)=p(t)+\int_{0}^{T} S(t, s) f(s, x(s)) d s, t \in[0, T], \text { for all } x \in X
$$

(E) There exists $x_{0} \in X$ such that

$$
\begin{gathered}
x_{0}(t) \leq p(t)+\int_{0}^{T} S(t, s) f\left(s, x_{0}(s)\right) d s \\
t \mid x_{0}(t)-\left[p(t)+\int_{0}^{T} S(t, s) f\left(s, x_{0}(s)\right) d s\right] .
\end{gathered}
$$

(F) $s \mid \int_{0}^{T} S(t, s)[f(s, y(s))-f(s, x(s))] d s$ for all $x, y \in X$ with $x \sim y$ and $s \in[0, T]$

We have the following result on the existence of solutions for integral equations.

Theorem 9. Under assumptions $(A)-(F)$, the integral Equation (42) has a unique solution in $X=C([0, T], R)$.
Proof. Let $H: X \longrightarrow X$ be defined by

$$
H x(t)=p(t)+\int_{0}^{T} S(t, s) f(s, x(s)) d s, t \in[0, T] \text {, for all } x \in X
$$

First, we will prove that $H$ is $E$-preserving. Let $x \sim y$. Then, we have $s \mid \int_{0}^{T} S(t, s) f(s, y(s)) d s-\int_{0}^{T} S(t, s) f(s, x(s)) d s$ for all $s \in[0, T]$. On the other hand, by the definition of $H$, we have

$$
H y-H x=\int_{0}^{T} S(t, s)[f(s, y(s))-f(s, x(s))] d s, \text { for all } t \in[0, T]
$$

So, $H x \sim H y$. 
Now, suppose that $x, y \in X$ with $x \sim y$. Then, by $(C),(D)$ and the definition of $H$, we get

$$
\begin{aligned}
P_{m}(H x, H y, \alpha) & =\sup _{t \in[0, T]} \frac{1}{1+\left(\alpha t^{2}\right)}|H x(t)-H y(t)| \\
& \leq \sup _{t \in[0, T]} \frac{1}{1+\left(\alpha t^{2}\right)} \int_{0}^{T} S(t, s)|f(s, x(s))-f(s, y(s))| d s \\
& \leq k\left(\frac{1}{1+\left(\alpha t^{2}\right)} \max \left\{|x(s)-y(s)|,|x(s)-H x(s)|,|y(s)-H y(s)|, \frac{|x(s)-H y(s)|+|y(s)-H x(s)|}{2}\right\}\right) \\
& \leq k\left(\frac{1}{1+\left(\alpha t^{2}\right)} \max \left\{\sup _{s \in[0, T]}|x(s)-y(s)|, \sup _{s \in[0, T]}|x(s)-H x(s)|, \sup _{s \in[0, T]}|y(s)-H y(s)|, \frac{\sup _{s \in[0, T]}|x(s)-H y(s)|+\sup _{s \in[0, T]}|y(s)-H x(s)|}{2}\right\}\right) \\
& \leq k\left[\max \left\{P_{m}(x, y, \alpha), P_{m}(x, H x, \alpha), P_{m}(y, H y, \alpha), \frac{P_{m}(x, H y, \alpha)+P_{m}(y, H x, \alpha)}{2}\right\}\right] .
\end{aligned}
$$

Now, by $(E)$, there exists $x_{0} \in X$ such that $x_{0} \sim H x_{0}$. Then, the conditions of Theorem 4 are satisfied and hence the integral Equation (42) has a unique solution in $X=C([$ $0, T], \mathbb{R})$. Note that $\alpha \equiv 1$ when $\ell \sim \ell^{\prime}$ and $\alpha \equiv 0$ when $\ell+\ell^{\prime}$.

\section{Conclusion}

This article presents fixed point theorems for mappings satisfying the contraction type conditions defined with auxiliary functions over $E$-parametric metric spaces. The readers will get many fixed point results as the outcome of this work. These results have been obtained in the following ways:

(1) Defining an auxiliary function through a particular form

(2) Equipping the $E$-parametric metric space $\left(H, E, P_{m}\right)$ with a directed graph and defining $\alpha(x, y)=1$, whenever $(x, y)$ is an edge in a directed graph defined on $H$ and $\alpha(x, y)=0$ otherwise. Similarly, we may consider a partial order on $H$ instead of a directed graph

(3) Defining an equivalence relation on $H$ by edge/path of a graph, a partial order, a preorder, etc.

(4) Taking $P_{m}\left(\ell, \ell^{\prime}, \vee\right)=d\left(\ell, \ell^{\prime}\right)$ for all $\vee$, where $d$ is used for a metric on $H$

\section{Data Availability}

No data were used to support this study.

\section{Conflicts of Interest}

The authors declare that they have no competing interests.

\section{Authors' Contributions}

All authors contributed equally and significantly in writing this article. All authors read and approved the final manuscript.

\section{References}

[1] P. Salimi, A. Latif, and N. Hussain, "Modified $\alpha$ - $\psi$-contractive mappings with applications," Fixed Point Theory and Applications, vol. 2013, 2013.

[2] H. Afshari, H. Aydi, and E. Karapinar, "On generalized $\alpha-\psi$ Geraghty contractions on b-metric spaces," Georgian Mathematical Journal, vol. 27, no. 1, pp. 9-21, 2020.

[3] S. Czerwik, "Contraction mappings in b-metric spaces," Acta Mathematica et Informatica Universitatis Ostraviensis, vol. 1, pp. 5-11, 1993.

[4] S. Czerwik, "Nonlinear set-valued contraction mappings in b-metric spaces," Atti del Seminario Matematico e Fisico dell'Università di Modena, vol. 46, pp. 263-276, 1998.

[5] E. Gilic, D. Dolicanin-Dekic, Z. D. Mitrovic, D. Pucic, and H. Aydi, "On some recent results concerning F-Suzukicontractions in b-metric spaces," Mathematics, vol. 8, p. 940, 2020.

[6] H. Aydi, A. Felhi, E. Karapinar, and S. Sahmim, "A Nadlertype fixed point theorem in dislocated spaces and applications," Miskolc Mathematical Notes, vol. 19, pp. 111-124, 2018.

[7] S. G. Matthews, "Partial metric topology," Proceedings of the 8th Summer Conference on General Topology and Applications, Annals of the New York Academy of Sciences, vol. 728, pp. 183197, 1994

[8] Z. Mustafa, J. R. Roshan, V. Parvaneh, and Z. Kadelburg, "Some common fixed point results in ordered partial bmetric spaces," Journal of inequalities and Applications, vol. 2013, no. 1, p. 26, 2013. 
[9] V. Parvaneh and Z. Kadelburg, "Extended partial b-metric spaces and some fixed point results," Univerzitet u Nišu, vol. 32, no. 8, pp. 2837-2850, 2018.

[10] S. Khalehoghli, H. Rahimi, and M. Eshaghi Gordji, "Fixed point theorems in $R$-metric spaces with applications," AIMS Mathematics, vol. 5, no. 4, pp. 3125-3137, 2020.

[11] N. Hussain, S. Khaleghizadeh, P. Salimi, and A. A. N. Abdou, "A new approach to fixed point results in triangular intuitionistic fuzzy metric spaces," Abstract and Applied Analysis, vol. 2014, Article ID 690139, 2014.

[12] M. U. Ali, T. Kamran, and W. Sintunavarat, "Fixed point theorems for multi-valued mappings with a lupphi function," Proceedings of the National Academy of Sciences, India Section A: Physical Sciences, vol. 88, no. 1, pp. 33-39, 2018. 